long disease duration. The other important finding of this study is the increased expression of membrane CD11b in fibrocytes. The integrin CD11b (also known as complement receptor type 3, macrophage antigen- 1 and $\alpha_{M} \beta_{2}$ ), which is primarily expressed on neutrophils, macrophages and monocytes, is critical for the normal function of these cells and plays an important role in the development of inflammatory responses. CD11b is expressed in a low-affinity, inactive conformation in circulating leukocytes, but is rapidly upregulated on the cell surface during stimulusdependent activation [10]. The factors triggering upregulation of $\mathrm{CD} 11 \mathrm{~b}$ in fibrocytes and its biological significance are not known. One hypothesis is that the increased expression of $\mathrm{CD} 11 \mathrm{~b}$ in fibrocytes reflects their activation in the course of the inflammatory response, as in patients with $\mathrm{PAH}$. As a consequence of CD11b upregulation, fibrocytes might display an increased capability to adhere to endothelial cells, extravasate and subsequently transmigrate into the surrounding interstitial tissue. Fibrocytes therefore can be proposed to play a critical role in the initiation and maintenance of inflammation in $\mathrm{PAH}$, even though they are not detected in the lung tissues. A central question that remains to be answered is whether fibrocytes differentiate into myofibroblasts, or whether they transiently stimulate differentiation and proliferation of other progenitor cells or resident mesenchymal cells within the lung.

In conclusion, our data suggest that the level of circulating fibrocytes may not be predictive of disease activity and progression, and more detailed analysis of their functional capacity and activation is needed. CD11b upregulation by circulating fibrocytes may have a role in the initiation and/or progression of $\mathrm{PAH}$ but detailed investigations on fibrocyte function will be required.

N. Gambaryan, S. Cohen-Kaminsky, D. Montani, B. Girerd, A. Huertas, A. Seferian, M. Humbert and F. Perros

Faculté de Médecine, Université Paris-Sud, Kremlin-Bicêtre, France. Centre de Référence de l'Hypertension Pulmonaire Sévère, Service de Pneumologie et Réanimation Respiratoire, Hôpital Antoine Béclère, Assistance Publique - Hôpitaux de Paris, Clamart, France. INSERM U999, Hypertension Artérielle Pulmonaire, Physiopathologie et Innovation Thérapeutique, Centre Chirurgical Marie Lannelongue, Le Plessis-Robinson, France. Département de la Recherche Médicale, Centre Chirurgical Marie Lannelongue, Le Plessis-Robinson, France.
Correspondence: F. Perros, INSERM U999, Centre Chirurgical Marie Lannelongue, 133 avenue de la Resistance, 92350 Le Plessis-Robinson, France. E-mail: frederic.perros@gmail.com

Support Statement: This study was supported by HTAP France.

Statement of Interest: Statements of interest for D. Montani and M. Humbert can be found at www.erj.ersjournals.com/site/ misc/statements.xhtml

\section{REFERENCES}

1 Stenmark KR, Frid MG, Yeager ME. Fibrocytes: potential new therapeutic targets for pulmonary hypertension? Eur Respir J 2010; 36: 1232-1235.

2 Nikam VS, Schermuly RT, Dumitrascu R, et al. Treprostinil inhibits the recruitment of bone marrow-derived circulating fibrocytes in chronic hypoxic pulmonary hypertension. Eur Respir J 2010; 36: 1302-1314.

3 Montani D, Perros F, Gambaryan N, et al. C-kit positive cells accumulate in remodeled vessels of idiopathic pulmonary arterial hypertension. Am J Respir Crit Care Med 2011; 184: 116-123.

4 Gambaryan N, Perros F, Montani D, et al. Targeting of c-kit+ haematopoietic progenitor cells prevents hypoxic pulmonary hypertension. Eur Respir J 2011; 37: 1392-1399.

5 Frid MG, Brunetti JA, Burke DL, et al. Hypoxia-induced pulmonary vascular remodeling requires recruitment of circulating mesenchymal precursors of a monocyte/macrophage lineage. Am I Pathol 2006; 168: 659-669.

6 Bucala R, Spiegel L, Chesney J, et al. Circulating fibrocytes define a new leukocyte subpopulation that mediates tissue repair. Mol Med 1994; 1: 71-81.

7 Strieter RM, Keeley EC, Hughes MA, et al. The role of circulating mesenchymal progenitor cells (fibrocytes) in the pathogenesis of pulmonary fibrosis. J Leukoc Biol 2009; 86: 1111-1118.

8 Pilling D, Vakil V, Gomer RH. Improved serum-free culture conditions for the differentiation of human and murine fibrocytes. J Immunol Methods 2009; 351: 62-70.

9 Heeschen C, Lehmann R, Honold J, et al. Profoundly reduced neovascularization capacity of bone marrow mononuclear cells derived from patients with chronic ischemic heart disease. Circulation 2004; 109: 1615-1622.

10 Hogg N, Leitinger B. Shape and shift changes related to the function of leukocyte integrins LFA-1 and Mac-1. J Leukoc Biol 2001; 69: 893-898.

\title{
Artificial lung as an alternative to mechanical ventilation in COPD exacerbation
}

\section{To the Editors:}

Acute exacerbation of chronic obstructive pulmonary disease (COPD) is commonly treated with different kinds of noninvasive positive pressure devices, ranging from helmet or face-mask continuous positive airway pressure (CPAP) to noninvasive pressure support ventilation (NPPV), or Bi-PAP [1].
The use of positive end-expiratory pressure (PEEP) and NPPV often results in the successful treatment of COPD patients with respiratory distress [1, 2]. If, despite maximal medical management, respiratory distress and gas exchange deteriorate with increasing tachypnoea and acidosis, and with altered level of consciousness, then tracheal intubation and mechanical 
ventilation (MV) become mandatory [3]. However, tracheal intubation and MV have several detrimental side-effects that may concur to determine the high morbidity and mortality reported in COPD patients requiring them [3,4]. Indeed, the increase in airway resistance, the prolonged time required for lung emptying and the resulting dynamic hyperinflation, named auto-PEEP [4], are the most important physiological alterations during COPD exacerbation. In this condition, the application of MV could increase lung hyperinflation and lead to barotrauma and circulatory failure $[4,5]$. Furthermore, tracheal intubation is usually associated with the need for sedation. The side-effects of intubation, sedation and MV may initiate a vicious circle, often resulting in a very difficult or impossible weaning.

In this report, we describe a patient with severe exacerbation of COPD, in whom after failure of noninvasive ventilation, we decided to treat the respiratory acidosis, tachypnoea and ventilatory fatigue by removing $\mathrm{CO}_{2}$ with an artificial lung. This avoided the need for tracheal intubation and MV, leaving the patient in spontaneous breathing.

In September 2010, a 72-yr-old, female heavy smoker with a history of COPD (forced expiratory volume in $1 \mathrm{~s}$ (FEV1) $50 \%$ predicted, forced vital capacity (FVC) $94 \%$ and Tiffeneau index $41 \%$ ) arrived at the emergency department with an acute COPD exacerbation. She had acute respiratory failure with severe respiratory acidosis ( $\mathrm{pH} 7.27$, carbon dioxide tension $79 \mathrm{mmHg}$ ) and tachypnoea $\left(40\right.$ breaths $\left.\cdot \mathrm{min}^{-1}\right)$, without any other organ dysfunction. A chest radiograph showed lung hyperinflation without signs of pneumonia. The patient was given standard medical treatment (antibiotics and bronchodilators) and steroids. After $96 \mathrm{~h}$ of NPPV, her respiratory acidosis further deteriorated (before extracorporeal membrane oxygenation; pre-ECMO in table 1). Tracheal intubation with MV was considered, but, as an alternative, we proposed to the patient and her son the use of an artificial lung, informing them of the possible advantages (and risks) of this approach, including the relief of dyspnoea, the natural lung deflation, the maintenance of consciousness and spontaneous breathing, without tracheal intubation. Thereafter, we applied a veno-venous bypass by a percutaneous cannulation of both femoral veins. The procedure was performed under mild sedation and local anaesthesia. The extracorporeal system consisted of the Permanent Life Support Set, "Bioline Coating", Maquet BE-PLS 2050® (Rotaflow RF 32 Pump and Quadrox PLS Oxygenator; Maquet GmbH, Rastatt, Germany).

The extracorporeal blood flow was set at $2 \mathrm{~L} \cdot \mathrm{min}^{-1}$ and maintained stable, while the gas flow, which is the main determinant of the extracorporeal $\mathrm{CO}_{2}$ removal, was increased slowly (starting from $1 \mathrm{~L} \cdot \mathrm{min}^{-1}$ ) to avoid huge and sudden alteration of the acid-base status (table 1). The patient was nonintubated and breathing a humidified air-oxygen mixture to maintain normal arterial oxygen tension $\left(\mathrm{Pa}_{\mathrm{a}} \mathrm{O}_{2} ;\right.$ table 1$)$. After $48 \mathrm{~h}$, the patient's metabolic $\mathrm{CO}_{2}$ production was almost totally cleared through the membrane lung $\left(4 \mathrm{~L} \cdot \mathrm{min}^{-1}\right.$ gas flow) (fig. 1a). With this setting, the artificial $\mathrm{CO}_{2}$ removal almost abolished the need to clear the $\mathrm{CO}_{2}$ through the natural lung. In fact, the patient's dyspnoea was relieved shortly after starting the bypass, and, when total extracorporeal $\mathrm{CO}_{2}$ removal was reached, the respiratory rate decreased from 32 to 8 breaths. $\min ^{-1}$ and the pleural pressure, as measured by tidal oesophageal pressure swings, an index of work of breathing, completely normalised (fig. 1b). Radiographic measurements on chest radiographs confirmed the progressive resolution of the dynamic hyperinflation (table 1).

We therefore began to slowly reduce the extracorporeal $\mathrm{CO}_{2}$ removal, allowing the patient to increase her minute ventilation,

TABLE 1 Patient's gas exchange and breathing pattern, radiographic measures of hyperinflation and artificial lung parameters

Pre-ECMO

On ECMO

$\begin{array}{cc}\text { Day } 6 \text { off } & \text { Day } 7 \\ \text { ECMO } & \text { discharge }\end{array}$

\begin{tabular}{|c|c|c|c|c|c|c|c|c|}
\hline & & & \multirow[b]{2}{*}{ 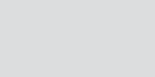 } & \\
\hline & & Day 1 & Day 2 & Day 3 & Day 4 & Day 5 & & \\
\hline Treatment & CPAP $\#$ & Face mask & Face mask & Face mask & Face mask & Face mask & Face mask & $\mathrm{Nasal} \mathrm{cannula}^{+}$ \\
\hline $\mathrm{pH}$ & 7.226 & 7.430 & 7.392 & 7.466 & 7.430 & 7.460 & 7.452 & 7.463 \\
\hline $\mathrm{PCO}_{2} \mathrm{mmHg}$ & 98.3 & 57.0 & 64.8 & 52.5 & 47,2 & 64 & 61.7 & 58.5 \\
\hline $\mathrm{PO}_{2} \mathrm{mmHg}$ & 71.1 & 79.5 & 122 & 77.4 & 77 & 98 & 74 & 68.7 \\
\hline MBDS & 10 & 7 & 2 & 3 & 3 & 2 & 1 & 1 \\
\hline CXR lung height $\mathrm{cm}$ & 29.8 & 26.6 & 26.6 & & 25.2 & & 24.1 & 25 \\
\hline CXR DAOD & 15 & 17 & 18 & & 20 & & 22 & 20 \\
\hline ECMO BF L. $\min ^{-1}$ & & 2.03 & 2.04 & 2.27 & 2.03 & 1.5 & 1.48 & \\
\hline ECMO GF L. $\min ^{-1}$ & & 3 & 4 & 2 & 0.9 & 0.4 & 0 & \\
\hline
\end{tabular}

ECMO: extracorporeal membrane oxygenation; $\mathrm{CPAP}$ : continuous positive airway pressure; $\mathrm{Fl}_{1} \mathrm{O}_{2}$ : inspiratory oxygen fraction; $\mathrm{PCO}_{2}$ : carbon dioxide tension; $\mathrm{PO}_{2}$ : oxygen tension; fR: respiratory frequency; $\triangle$ Poes: change in intratidal oesophageal pressure; MBDS: modified Borg dyspnoea scale; CXR: chest radiograph; DAOD: diaphragmatic angle (both measured in the right lung, according to [6]); BF: blood flow; GF: gas flow. ${ }^{\#:} 12 \mathrm{cmH}_{2} \mathrm{O}$; $: 10 \mathrm{~L} \cdot \mathrm{min}^{-1} \mathrm{O}_{2 ;}{ }^{+}: 4 \mathrm{~L} \cdot \mathrm{min}^{-1} \mathrm{O}_{2}$. 

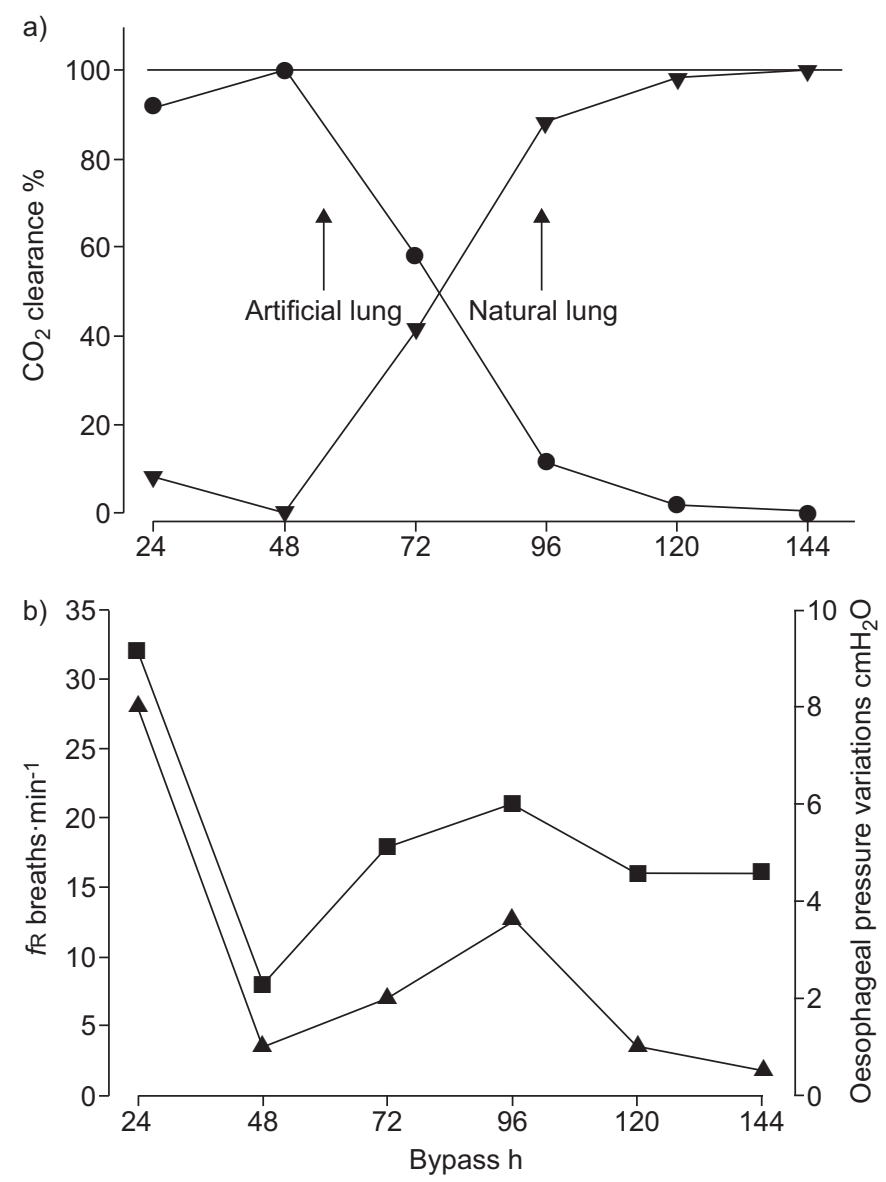

FIGURE 1. a) Percentage $\mathrm{CO}_{2}$ clearance, performed by the artificial lung and the estimated $\mathrm{CO}_{2}$ clearance of the natural lung as a function of the bypass time. b) Respiratory frequency $f R(\boldsymbol{\square})$ and tidal oesophageal pressure variations $(\mathbf{\Lambda})$ as a function of bypass time. The above variables were unavailable during the first $24 \mathrm{~h}$ because the patient clinical conditions did not allow insertion of invasive monitoring

while monitoring respiratory rate, respiratory pleural pressure variations and breathing coordination. On day 6 , we removed the artificial $\mathrm{CO}_{2}$ removal and the patient showed an acceptable respiratory pattern and gas exchange without clinical and radiological signs of hyperinflation (table 1). After a few hours, the extracorporeal circuit was removed. The patient was discharged from the intensive care unit 1 day later, and at 6 months follow-up she was well and had no need of additional oxygen.

Since 1980, extracorporeal $\mathrm{CO}_{2}$ removal has been proposed to provide lung rest by reducing mechanical ventilation load in acute respiratory distress syndrome [7]; it has also been used, in combination with tracheal intubation and MV, to resolve acute asthma attacks [8] and dynamic hyperinflation [9]. Recently, this technique has been used in spontaneously breathing patients with the purpose of bridging to lung transplantation [10, 11].

We report herein the successful use of the extracorporeal $\mathrm{CO}_{2}$ removal in a nonintubated, spontaneously breathing patient to treat acute respiratory failure and dynamic hyperinflation during COPD exacerbation. Our primary aim was to aid recovery from dynamic hyperinflation while avoiding tracheal intubation and MV with its associated side-effects, such as the increase in hyperinflation, barotrauma, ventilatory-acquired pneumonia, critically polyneuropathy illness and difficult weaning $[2,3]$. The improvement of the extracorporeal biocompatible materials and circuit characteristics (i.e. new centrifugal pumps, high-performance artificial lungs and heparincoated circuits) reduces the risks (mainly haemorrhage) of this invasive technique, which could become a possible alternative to conventional mechanical ventilation.

Starting the extracorporeal $\mathrm{CO}_{2}$ removal in this awake and spontaneously breathing patient almost immediately produced a new clinical scenario. The need of breathing was sharply reduced, proportionally to the $\mathrm{CO}_{2}$ cleared by the membrane lung, the respiratory muscles were at rest and the lungs deflated naturally. Despite her severe respiratory condition, the patient was able to communicate, drink and eat spontaneously. In this condition, the relationship between the patient and the intensive care unit team (medical doctors and nurses) had to be completely modified, since the patient's needs were totally different from those of sedated mechanically ventilated patients.

In this awake nonintubated patient, the approach to the monitoring of the usual respiratory parameters was particularly difficult. In fact, tidal volume, FEV1 and FVC measurements were attempted using a mouthpiece spirometer, but were too affected by patient cooperation and ventilatory fatigue to speculate on. Furthermore, auto-PEEP and mixed expired $\mathrm{CO}_{2}$ could not be directly measured. We recorded the patient oxygen consumption and the extracorporeal oxygen supply using the Fick principle, the extracorporeal $\mathrm{CO}_{2}$ clearance, measuring the $\mathrm{CO}_{2}$ concentration in the sweep gas flow, and derived the patient $\mathrm{CO}_{2}$ clearance assuming a respiratory quotient equal to 1. We also recorded the blood gas parameters, the respiratory mechanical coordination, the modified Borg dyspnoea scale and the intra-tidal oesophageal pressure swings, as an index of the work of breathing. Although some of these parameters were approximate, we believe that their comprehensive evaluation was very useful to understand the mechanisms involved in the vicious circle of dynamic hyperinflation and to guide the appropriate use of the extracorporeal $\mathrm{CO}_{2}$ removal. Daily chest radiographs helped us to confirm the recovery from dynamic hyperinflation. Indeed, more accurate and precise noninvasive methods to monitor a spontaneous breathing patient during extracorporeal treatment would be highly desirable.

In conclusion, in patients with acute severe COPD exacerbation who fail the noninvasive ventilation approach, the use of veno-venous extracorporeal $\mathrm{CO}_{2}$ removal during spontaneous breathing could be a successful strategy to reduce dynamic hyperinflation and to avoid the detrimental effects of tracheal intubation and mechanical ventilation.

\section{S. Crotti*, A. Lissoni*, D. Tubiolo*, S. Azzari", P. Tarsia ", L. Caspani* and L. Gattinoni*,\#}

*Dipartimento di Anestesia, Rianimazione (Intensiva e Subintensiva) e Terapia del Dolore, Fondazione IRCCS Ca' Granda Ospedale Maggiore Policlinico di Milano, "Dipartimento di Anestesiologia, Terapia Intensiva e Scienze Dermatologiche, Università degli Studi, and "Dipartimento Toracopolmonare e 
Cardiovascolare, Università di Milano, Fondazione IRCCS Ca' Granda Ospedale Maggiore Policlinico di Milano, Milan, Italy.

Correspondence: S. Crotti, Terapia Intensiva "E. Vecla", Dipartimento di Anestesia, Rianimazione e Terapia del Dolore; Fondazione IRCCS Ca' Granda Ospedale Maggiore Policlinico, Via F. Sforza 35, 20122, Milano, Italy. E-mail: stefania.crotti@ policlinico.mi.it

Statement of Interest: None declared.

Acknowledgements: The authors would like to thank the entire intensive care unit staff (nurses and doctors) who fully managed veno-venous extracorporeal support and who have earned the credit for the successful and uneventful course described.

\section{REFERENCES}

1 Nava S, Hill N. Non-invasive ventilation in acute respiratory failure. Lancet 2009; 374: 250-259.

2 Plant PK, Owen JL, Elliott MW. A multicentre randomised controlled trial of the early use of non-invasive ventilation in acute exacerbation of chronic obstructive pulmonary disease on general respiratory wards. Lancet 2000; 355: 1931-1935.
3 Ward NS, Dushay KM. Clinical concise review: mechanical ventilation of patients with chronic obstructive pulmonary disease. Crit Care Med 2008; 36: 1614-1619.

4 Raghu MR, Kalpalatha KG. Review of ventilator techniques to optimize mechanical ventilation in acute exacerbation of chronic obstructive pulmonary disease. Int J COPD 2007; 2: 441-452.

5 Budweiser S, Jörres RA, Pfeifer M. Treatment of respiratory failure in COPD. Int J COPD 2008; 3: 605-618.

6 Rothpearl A, Varma AO, Goodman K. Radiographic measures of hyperinflation in clinical emphysema. Discrimination of patients from controls and relationship to physiologic and mechanical lung function. Chest 1988; 94: 907-913.

7 Gattinoni L, Pesenti A, Mascheroni D, et al. Low-frequency positive-pressure ventilation with extracorporeal $\mathrm{CO}_{2}$ removal in severe acute respiratory failure. JAMA 1986; 256: 881-886.

8 Mikkelsen ME, Woo J, Sager JS, et al. Outcomes using extracorporeal life support for adult respiratory failure due to status asthmaticus. ASAIO J 2009; 55: 47-52.

9 Cardenas VJ, Lynch JE, Ates R, et al. Venovenous carbon dioxide removal in chronic obstructive pulmonary disease. Experience in one patient. ASAIO J 2009; 55: 420-422.

10 Strueber M. Extracorporeal support as a bridge to lung transplantation. Curr Opin Crit Care 2010; 16: 69-73.

11 Olsson KM, Simon A, Strueber M, et al. Extracorporeal membrane oxygenation in nonintubated patients as bridge to lung transplantation. Am J Transplant 2010; 10: 1-6.

DOI: $10.1183 / 09031936.00021111$

\section{Intermittent hypoxia enhances cancer progression in a mouse model of sleep apnoea}

\section{To the Editors:}

Obstructive sleep apnoea (OSA) is a very prevalent syndrome that induces or aggravates cardiovascular, metabolic and neurocognitive disorders. Among the various challenges imposed on a patient by OSA as a consequence of apnoeas (sleep disruption, increased inspiratory efforts and recurrent hypoxaemia), intermittent hypoxia plays a major role in the pathophysiology of this sleep breathing disorder.

It has been well established that hypoxia plays an important role in regulating the various stages of tumour formation and progression [1]. Accordingly, the aim of the experimental study reported here was to test the hypothesis that high-rate intermittent hypoxia with a time course similar to that found in OSA (one or more hypoxic event per minute) enhances tumour growth. To avoid the interaction of any comorbidity, this investigation was carried out in a well-controlled animal model where the main variable under study was intermittent hypoxia.

This study, which was approved by the Ethical Committee for Animal Research of the University of Barcelona (Barcelona, Spain), was conducted in 15 pathogen-free male C57BL/6J mice (25-30 g) using a conventional murine melanoma model consisting of tumour induction by subcutaneous injection of $10^{6}$ B16F10 melanoma cells (ATCC-CRL-6475; American Type Culture Collection, Manassas, VA, USA) in the left flank region of the mouse [2]. This is a widely used cancer model in experimental research $[3,4]$, which has a response to hypoxia that is representative of a variety of cancer types $[5,6]$.

Application of chronic intermittent hypoxia (60 events $\cdot h^{-1}$ consisting of $20 \mathrm{~s}$ of $5 \% \mathrm{O}_{2}$ followed by $40 \mathrm{~s}$ of room air) started on the same day as the injection of cancer cells. Briefly, a continuous flow of gas was circulated through a box $(26 \mathrm{~cm}$ long, $18 \mathrm{~cm}$ wide and $6 \mathrm{~cm}$ high) by means of a distribution system based on multiple orifices. A pneumatic valve placed near the inlet of the box cyclically switched from the room air entrance (40 s) to a gas reservoir of hypoxic air at an oxygen fraction of $5 \%(20 \mathrm{~s})$. Seven mice were placed in the intermittent hypoxia box, with food and water available, for $6 \mathrm{~h} \cdot \mathrm{day}^{-1}$ during the light period (10:00-16:00 h) for 14 consecutive days. A normoxia control group of eight mice was placed in an identical system with the reservoir of $5 \% \mathrm{O}_{2}$ air replaced by room air. Accordingly, both groups of melanoma-injected mice were subjected to exactly the same protocol, the only difference being the breathing of normoxic or intermittently hypoxic air.

The growth of the melanoma tumour was assessed at days 8, 11 and 14 after the injection of cancer cells by externally measuring the tumour's dimensions with a calliper and estimating tumour volume as $W^{2} \times L / 2$, where $W$ is tumour width and $L$ is tumour length [2]. At day 14, the mice were sacrificed and the tumours were excised and weighed to directly measure cancer growth. 